\title{
Exploiting 2-D to 3-D Intra-Operative Image Registration for Qualitative Evaluations and Post-Operative Simulations
}

\author{
André Guéziec ${ }^{1 \star}$, Kenong $\mathrm{Wu}^{2}$, Bill Williamson ${ }^{2}$, Peter Kazanzides ${ }^{2}$, \\ Robert Van Vorhis ${ }^{2}$, and Alan Kalvin ${ }^{3}$ \\ 1 Multigen-Paradigm \\ 550 S. Winchester Bd., Suite 500, San Jose, CA 95128 \\ 2 Integrated Surgical Systems \\ 1850 Research Park Drive, Davis, CA 95616 \\ 3 IBM T.J. Watson Research Center \\ 30 Sawmill River Road, Hawthorne, NY 10532, USA
}

\begin{abstract}
This paper addresses a key issue of providing clinicians with visual information to validate the accuracy of $2 \mathrm{D} / 3 \mathrm{D}$ registration for robot-assisted total hip replacement (THR) surgery. In practice, clinicians rely on post-operative X-rays to assess the accuracy of implant placement. Motivated by this, we simulate a set of post-operative X-ray images by superimposing the implant positioned pre-operatively onto the intra-operatively collected and calibrated images of the femur, through a transformation computed by the $2 \mathrm{D} / 3 \mathrm{D}$ registration. With these images, a judgment on the registration accuracy can be made. In addition, this paper introduces methods for superimposing pre-operative data on intra-operative X-ray images that were not corrected for distortion, by applying the same image distortion to the data. This paper also introduces a new framework for incorporating surface normals in the objective function for registration. A comparison between marker-based and image-based registration is conducted.
\end{abstract}

Keywords: Simulation of post-operative X-ray images, Anatomy- and Image-Based Registration, Total Hip Replacement Surgery, Robodoc ${ }^{\mathrm{R}}$, CT, X-ray Fluoroscopy

\section{Introduction}

In this paper, we address a key issue of providing clinicians with visual information for determination of accuracy of the image-based registration for robot-assisted surgery. Although numerous registration approaches have been presented, the topic of registration validation has scarcely been addressed in the

\footnotetext{
* A. Guéziec is also a consultant in Medical Imaging based in the Silicon Valley.E-mail: gueziec@computer.org. This work was done while the first author was with IBM. 
literature. This is vital however for the acceptance of image-based registration and image guidance of therapy in practice.

This work is part of a joint study with Integrated Surgical System (ISS), of Davis, CA, to extend the RoBodoc ${ }^{\mathrm{R}}$ system [1]. Used for Total Hip Replacement (THR) surgery, the surgical robot of the RoBoDoC ${ }^{\mathrm{R}}$ system accurately mills the cavity for the femoral implant. The robot trajectory is planned using the ORTHODOC ${ }^{\mathrm{R}}$ software, based upon a CT-scan of the hip and a CAD model of an appropriate femoral implant. To register the surgical robot to its planned trajectory, the basic clinical protocol uses two or three metallic markers. These markers are surgically inserted in the femur [2,3]. They are pre-operatively imaged with CT and located by the ORTHODOC ${ }^{\mathrm{R}}$ software. Intra-operatively, they are located by the robot. With their positions in both CT and robot coordinate systems, a transformation between these two coordinate systems can be easily computed. This marker-based registration produces very reliable results and has been performed on more than 4,000 patients. However, marker insertion requires an additional surgery and sometimes causes discomfort for patients. We note that a CT-to-robot registration for robotic THR based on a proprietary technology (DigiMATCH ${ }^{\mathrm{TM}}$ ) without using markers was developed at ISS and used in clinics recently. The approach reported in this paper was developed independently and is different from DiGiMATCH ${ }^{\mathrm{TM}}$.

One of our goals is to study the feasibility of substituting external markerbased registration with X-ray image-based registration ${ }^{1}$. After a registration is performed, the following question is always raised: Is the computed transformation accurate enough to guide surgery? If the answer is yes, the surgeon will execute the robot surgery. Otherwise, another registration must be performed. Our approach is aimed at providing surgeons with simulated post-operative Xray images to assess registration accuracy.

In this paper, we introduce new developments of a method for registering CTscan images acquired pre-operatively to intra-operative X-ray images [4]. This registration allows us to accurately associate a surgical plan with the current pose of the surgical robot and the patient inside the operating room. To do this, we first extract the surface model of the femur from the CT image. Then, we acquire a few X-ray images from different points of view intra-operatively and compute projection lines for all images. Finally, we compute the transformation between CT space and robot space by matching the projection lines to the contours of the femur surface. Since the registration accuracy depends critically on this matching process, we introduce in Section 3 a new improvement: we refine the matches by exploiting the correlation between surface normals measured from surface models and surface normals estimated from 2-D X-rays using projection lines.

1 In addition to external markers, anatomical features, such as bone surfaces, can also be used for registration, which is usually called anatomy-based registration. The image-based registration in this paper is a particular type of anatomy-based registration. 


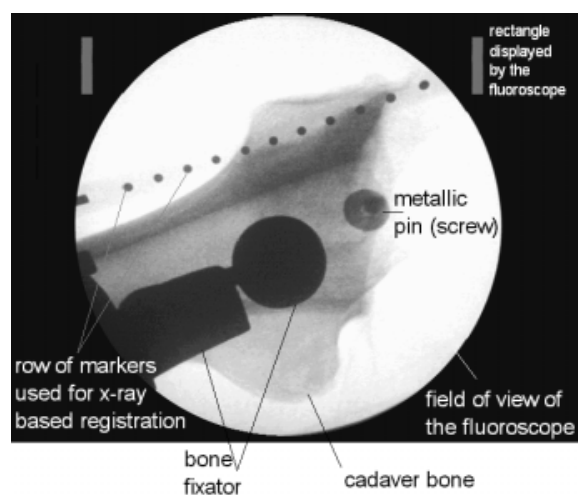

A

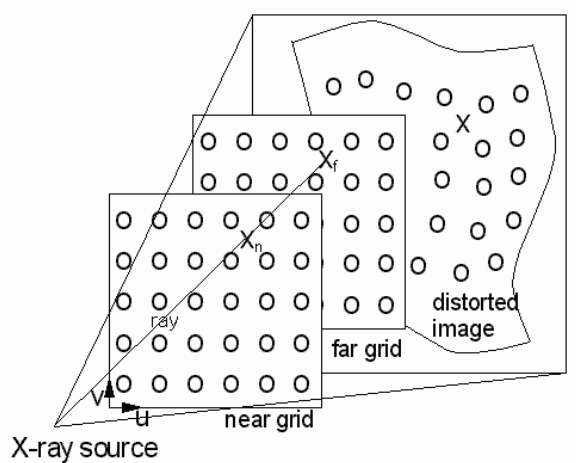

$B$

Fig. 1. A: Characteristics of typical X-ray images in this study: limited field of view, geometric distortion and occlusion (dots are used for calibration). B: Multi-grid calibration method. Any image pixel $X$ can be associated to two locations $X_{f}$ and $X_{n}$ on the grids, specifying a ray in three-dimensional space.

In Section 5, using an X-ray camera calibrated with respect to physical space and a registration of pre-operative data to physical space, we can effectively integrate various parts of the pre-operative data onto intra-operative X-ray images, as if the data had been part of the scene captured by the X-ray camera. This can be performed accurately, with or without correcting images for distortion, depending on which images the clinician is more comfortable with. In this way, we can, for instance, simulate post-operative X-rays.

\section{X-Ray Camera Calibration [4]}

We have attempted to work with X-ray images that reflect the data that can realistically be acquired during intra-operative X-ray fluoroscopy. The characteristics of these images are as follows: limited field of view (typically 6 inches and sometimes 9), geometric distortion, and in particular, occlusion of anatomical structures by various metallic instruments (see Fig. 1-A). Assuming that direct digital acquisition of X-rays will at some point supersede image intensifiers, intra-operative X-ray image quality should improve significantly, and the results of this paper correspond to a worst-case X-ray image quality.

The goal of camera calibration (X-ray, or other) is to determine a set of geometric parameters characterizing how a three-dimensional scene was projected onto the image. A perspective projection provides a suitable model for an X-ray camera. In addition, intra-operative X-rays are typically corrupted with two main types of distortion : "pincushion" distortion, and "S-shaped" distortion caused by interactions with stray magnetic fields during the image intensification process. This distortion must be compensated for before determining perspective projection parameters. Conventional methods would operate 
in two steps, by first correcting for the distortion and then determining the projection parameters using distortion-corrected images. Generally, both steps are accomplished by imaging grids of markers, whose position and orientation are precisely known with respect to the coordinate system that is relevant for calibration $[5,6,7,8,9,10,11]$.

The method that we retained combines distortion correction and geometric calibration in a single step, and extends the methods by Martins et al. [12] and by Champleboux et al. [13]: for each pixel of a digitized X-ray image, we determine directly the equation of a ray in three dimensional space that projected to that pixel. Knowledge of two points along the ray is sufficient for determining a ray. As illustrated in Fig. 1-B, the method works by imaging two grids, a "near" grid and a "far" grid, and observing the distorted grid points on the X-ray image. The grids are preferably observed by capturing separate images, and can even be "virtual", by moving a linear probe and collecting multiple images [4]. Knowing the correspondence between observed and physical grid points, for each pixel $X$ of the distorted image, it is possible to match it with a location on both grids ( $X_{n}$ on the near grid and $X_{f}$ on the far grid) by interpolation (preferably using thin-plate spline functions: we refer to [4] for a mathematical formulation). Both locations $X_{n}$ and $X_{f}$ in three-dimensions specify the ray that was sought for.

\section{2-D TO 3-D Registration Using Normals}

The new framework for handling normals that we are proposing could potentially be incorporated in other 2-D to 3 -D registration methods $[14,15,16,17,18]$.

The principle of the method that we built upon is as follows [4]: when the pre-operative femur model is perfectly registered with respect to physical space (surgical robot and X-ray images), X-ray paths (see Figs. 2) corresponding to the bone contours in the two-dimensional X-ray images and determined using image calibration information are tangent to (grazing) the surface of the femur model. Since the femur model is expected to be mis-registered in the beginning of the process, X-ray paths are a-priori not grazing (but intersecting, or "missing" the femur model altogether), and the registration process determines a rigid transformation optimizing the position and orientation of the surface model such that as many X-ray paths as possible become tangent to the surface. Figure 2 shows an experimental registration result: the position of the pre-operative bone model is gradually optimized until a large majority of the X-ray paths become tangent to it. The dark three-dimensional curves on the femur model of Figs. 2-E,F are silhouette curves. Silhouette curves, or "apparent contours", are an important component of this registration method: the method attempts to bring each X-ray path in contact with a silhouette curve. Given a center of perspective projection determined by calibration, silhouette curves are such that rays emanating from the center of perspective and tangent to the surface meet the surface on a silhouette curve.

To determine the rotation and translation that minimizes the sum of weighted squared distances between the silhouette curve points and the X-ray paths, we 


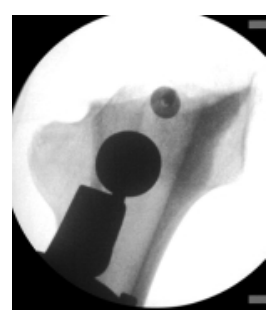

A

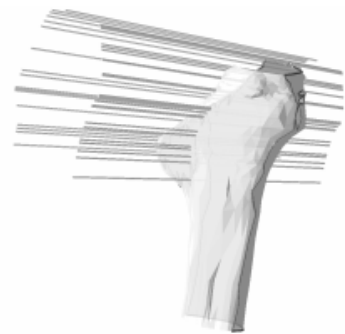

E

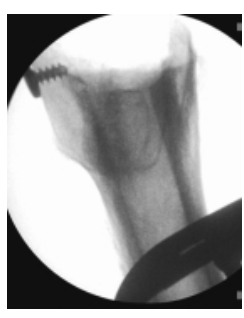

$B$

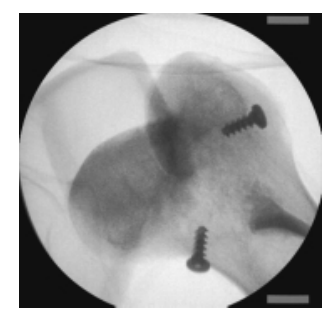

C

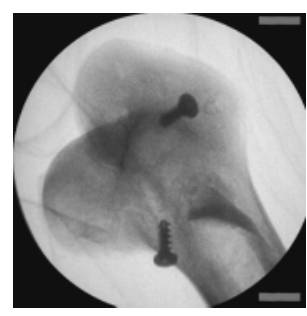

D

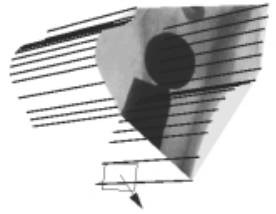

G

Fig. 2. A,B,C,D: four fluoroscopic views used for marker-less 2-D to 3-D imagebased registration. E,F: Pre-operative proximal femur model before (E) and after (F) registration to three-dimensional X-ray paths (straight lines) starting from the intra-operative X-ray source and going through bone contours in the X-ray images. The dark three-dimensional curves on the femur models are silhouette curves (depending on the position). G: pre-operative model in registered position with a distortion corrected X-ray image. Two X-ray paths taken consecutively along the bone contour define a normal vector shown in black. At the location where the (continuation of) an X-ray path is tangent to the bone surface, the surface normal (shown in white) should be aligned with the black normal vector.

use the following formulation:

$$
\min _{\mathbf{Q}, \mathbf{t}} \sum_{i=1}^{m} d_{i}^{2}=\min _{\mathbf{Q}, \mathbf{t}} \sum_{i=1}^{m}\left\|\mathbf{V}_{\mathbf{i}}\left(\mathbf{c}_{\mathbf{i}}-\left(\mathbf{Q p}_{\mathbf{i}}+\mathbf{t}\right)\right)\right\|^{2}, \mathbf{V}_{\mathbf{i}}=\left[\begin{array}{ccc}
0 & -v_{3} & v_{2} \\
v_{3} & 0 & -v_{1} \\
-v_{2} & v_{1} & 0
\end{array}\right]
$$

where $\mathbf{p}_{\mathbf{i}}$ are silhouette curve points (on the three-dimensional bone surface), $\mathbf{v}_{\mathbf{i}}$ are the unit direction vectors of the lines, $\mathbf{c}_{\mathbf{i}}$ are the positions of the center of perspectives corresponding to the lines (in this way, several views with several centers of perspective can be accommodated simultaneously), $d_{i}$ distances between straight lines and silhouette curve points, and $\mathbf{Q}$ and $\mathbf{t}$ are the unknown rotation matrix and translation vector. We use the Cayley rotation parameterization, which states that if $\mathbf{U}$ is a skew symmetric matrix obtained from a vector $\mathbf{u}$, then the matrix $\mathbf{Q}=(\mathbf{I}-\mathbf{U})(\mathbf{I}+\mathbf{U})^{-\mathbf{1}}$ is a rotational matrix. The advantage of this form is that for small rotations, $\mathbf{Q} \sim \mathbf{I}-\mathbf{2} \mathbf{U}$. The problem becomes:

$$
\min _{\mathbf{u}, \mathbf{t}} \sum_{i}\left\|\mathbf{V}_{\mathbf{i}}\left(\mathbf{p}_{\mathbf{i}}-\mathbf{c}_{\mathbf{i}}+2 \mathbf{P}_{\mathbf{i}} \mathbf{u}+\mathbf{t}\right)\right\|^{2}
$$


where $\mathbf{P}_{\mathbf{i}}$ is a skew symmetric matrix derived from $\mathbf{p}_{\mathbf{i}}$. Following Kumar et al. (1994) [19], we use the Tukey weighting function to scale each distance error, allowing the registration to operate in a fashion that is robust to outliers (incorrectly matched pairs: line, point). After some additional computations detailed in [4], we obtain a linear system that we solve for the desired transformations.

This formulation, and the registration accuracy, depend upon an algorithm to discover meaningful (lines, surface points) correspondences (although the robust formulation automatically ignores some erroneous correspondences). One strategy is to find the closest silhouette curve point from each straight line. In [4] the closest point was found by first decomposing each three-dimensional silhouette curve as a hierarchy of nested polygonal segments, each associated with a volume bounding the corresponding silhouette curve portion. Using this hierarchical description, a closest point can be queried in time proportional to the logarithm of the number of vertices in a silhouette curve.

In this paper we propose a general framework to improve the (lines, surface points) correspondences using information on normals. As illustrated in Fig 2$\mathrm{G}$, two X-ray paths taken consecutively along the bone contour define a normal vector. At the location where (the continuation of) an X-ray path is tangent to the bone surface, the surface normal should be aligned with the normal to the $\mathrm{X}$-ray path. This allows us to define constraints on the silhouette curve points that can be matched to the X-ray path, by considering matches for which the angle between the above-defined normals does not exceed a maximum value.

One method for incorporating surface normals in a projective registration process is described in Feldmar et al. [16]. Feldmar et al. develop an iterative closest point approach, wherein the distance is a (weighted) compromise between a three-dimensional distance and difference in normal orientation. Although this method succeeds in using surface normal information, since both position and normal terms are (squared, and then) added, one can compensate for the other: there is no difference between an erroneous positional match but correct normal match, a correct positional match but erroneous normal match or an incorrect match of both.

The approach we advocate is quite different and novel. The idea is to continue looking for a closest point in a usual (Cartesian) sense but also impose some constraints on the difference in normal orientations at the same time, essentially pruning out irrelevant curve portions. This is justified as follows: using the closest point is particularly relevant when we get very close to the ideal registration, since the exact match is a closest point with zero distance (modulo feature detection and calibration accuracy). Conversely, using normal orientation is better justified at the early stages of the registration to avoid local minima. We have observed for our data that although the normal orientation is generally correct, there is some question about its accuracy relative to the positional accuracy.

The algorithm used to implement the normal constraints can be better understood by examining Fig. 3. A silhouette curve (A) is mapped in (B) to the Gaussian sphere (unit sphere centered at the origin of Cartesian space) of normals by connecting the vertex normals of the surface model corresponding to 


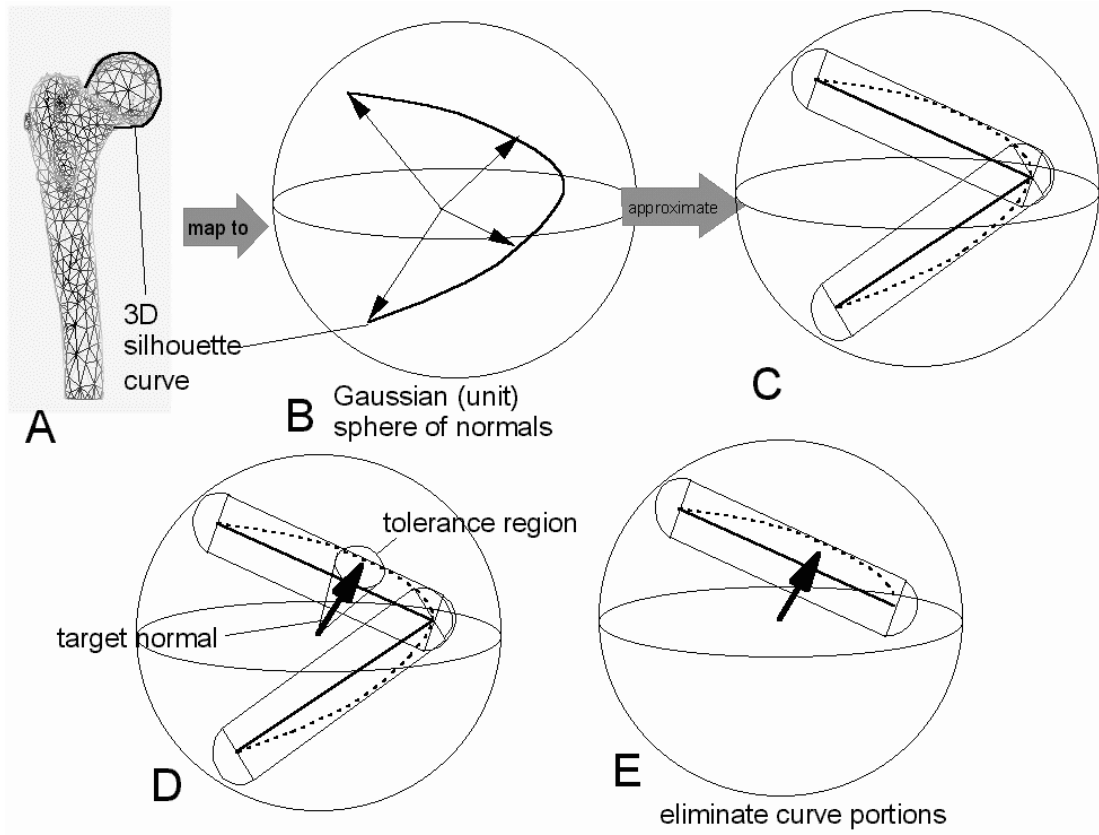

Fig. 3. A 3-D silhouette curve extracted from a surface model in (A) is mapped to the Gaussian sphere of normals in (B), is approximated by line segments in $(\mathrm{C})$, keeping accurate bounding volumes. Curve portions whose bounding volumes do not intersect the target normal (D) are unlikely matches and thus eliminated during the search for the closest point.

the vertices of the silhouette curve. A hierarchical description of this mapped curve is built $(\mathrm{C})$ in parallel to the hierarchical description in Cartesian space depicted above. For each X-ray path, a target normal (D) is determined as explained above and illustrated in Fig 2-C, with an associated tolerance region. Since the hierarchical description has segments with bounding areas, if a given bounding area (defined by a cylinder capped with two half spheres) does not intersect the tolerance region of the target normal (E), this means that this normal cannot possibly occur along the corresponding portion of silhouette curve, and thus the entire portion should not be considered for finding a closest point.

The algorithm explores the hierarchical curve description and decides to examine further a branch of the hierarchy (corresponding to a curve portion) only if the test on normals described above is passed. The rest of the algorithm is the same as before. Utilizing this new method has improved results in all the practical cases that we encountered as described next.

\section{Quantitative Evaluation of the Registration}

We used implanted fiducials on a cadaver bone and RoBodoc ${ }^{\mathrm{R}}$ to obtain precise and reliable transformations that can be compared to the image-based method. 
We CT-scanned the cadaver bone, then brought it to the operating room and fixated it to the robot. We then captured sets of X-ray fluoroscopy images and calibrated them geometrically with respect to the robot. We registered a CTbased model of the bone to the X-ray images. The results were compared with the marker-based technique. Starting with the registered position computed using the markers, we perturbed it with various rigid transformations, whose rotational axis was chosen using a random vector generator and whose rotation magnitude was fixed to three and five degrees. The translational component of the transformation was computed so as to leave the centroid of the markers invariant by the perturbation (any suitable point inside the bone could have been used), and simulate an initial position that could potentially occur in practice. The registration was completed in about 6 seconds on a UNIX workstation.

In clinical practice, orthopedic surgeons commonly examine the placement accuracy in terms of implant positions. We examined the registration accuracy by comparing the registered implant positions in the robot coordinate system. Specifically, we compared the displacements at the implant origin and tip as well as the rotation angle around the $Z$ axis of the implant coordinate system. This comparison is illustrated in Fig. 4. The implant coordinate system is shown in Fig. 4-A. The positions in the robot space transformed by the marker-based and image-based registrations are shown in Fig. 4-B. $d_{0}$ and $d_{t}$ are the displacements at the implant origin and tip, respectively.

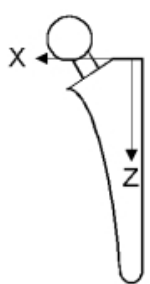

(A)

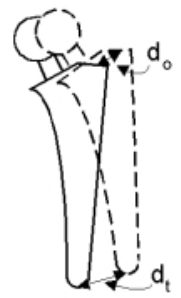

(B)

Fig. 4. Comparing implant positions. (a) Implant coordinate axes. (b) Implant positions in robot space: the implant transformed using the marker-based registration result is shown using a solid line while the implant transformed using the image-based registration result is shown using a dashed line.

Table 1 shows the comparison results. The average and maximum of absolute displacements at the implant tip and origin are $0.49 \mathrm{~mm}$ and $1.053 \mathrm{~mm}$, respectively. The average and maximum of the $\mathrm{Z}$ rotation errors are 2.64 and 4.55 degrees, respectively. According to our experience with surgeons, the requirements for displacement errors are approximately $1.5 \mathrm{~mm}$, and approximately 3 degrees for the rotation about the $\mathrm{Z}$ axis. ${ }^{2}$. Thus, assuming that the marker-based re-

\footnotetext{
${ }^{2}$ These criteria are derived by evaluating post-operative X-ray films. The exact values vary slightly among surgeons, and are difficult to evaluate. Note that we treat the marker-based registration results as the ideal results since we do not know the ideal answers.
} 
sults are the gold standard for registration, the accuracy in displacement achieves the clinical requirements in all cases while the rotational accuracy achieves the requirements in seven out of ten cases.

The reason for obtaining less accurate $\mathrm{Z}$ rotation parameters is the following. Since the shape of the femur is extremely elongated in the $\mathrm{Z}$ direction, the rotation change around the $\mathrm{Z}$ axis is much more sensitive to the same amount of distance mismatching than around the $\mathrm{X}$ and $\mathrm{Y}$ axes. This is also the reason why the normal constraints improve the registration accuracy. There are a few ways that the $\mathrm{Z}$ rotation accuracy can be improved. For example, we can choose better views for X-ray acquisition (different surgical approaches allow collection of different views), start with a better initial estimate, obtain more accurate femur surface models or give additional weight to particular measurements in the objective function.

\begin{tabular}{|l||c|c|c||c|c|c||c|}
\hline \multicolumn{9}{|c|}{ displacements and rotation in the implant coordinate system } \\
\hline & \multicolumn{2}{|c|}{ Origin displacement } & \multicolumn{3}{c||}{ Tip displacement } & Rotation \\
around Z \\
\hline Perturbation & $\mathrm{x}$ & $\mathrm{y}$ & $\mathrm{z}$ & $\mathrm{x}$ & $\mathrm{y}$ & $\mathrm{z}$ & \\
\hline rotation 3 degrees 1 & 0.975891 & 0.015485 & 0.565460 & 0.855482 & 0.414210 & 0.565020 & 1.979850 \\
\hline rotation 3 degrees 2 & 0.830098 & -0.489192 & 0.163470 & 0.534467 & -0.114655 & 0.162864 & 3.128288 \\
\hline rotation 3 degrees 3 & 0.982567 & -0.221832 & 0.271217 & 0.687081 & 0.115406 & 0.270682 & 2.688964 \\
\hline rotation 3 degrees 4 & 0.819053 & -0.357789 & 0.247661 & 0.620627 & 0.021679 & 0.247200 & 2.796623 \\
\hline rotation 3 degrees 5 & 0.941361 & 0.243733 & 0.672319 & 1.052943 & 0.678233 & 0.671715 & 0.976155 \\
\hline rotation 5 degrees 1 & 0.810275 & 0.411480 & -0.833420 & 0.658433 & 0.429188 & -0.833468 & -0.097202 \\
\hline rotation 5 degrees 2 & 0.848678 & -0.348916 & 0.222854 & 0.628410 & 0.015002 & 0.222230 & 2.846277 \\
\hline rotation 5 degrees 3 & 1.003069 & -0.685573 & -0.152810 & 0.351603 & -0.509426 & -0.154009 & 4.449264 \\
\hline rotation 5 degrees 4 & 0.843969 & -0.381169 & 0.258526 & 0.573869 & -0.006363 & 0.258017 & 2.872721 \\
\hline rotation 5 degrees 5 & 1.009851 & -0.774564 & -0.165820 & 0.432842 & -0.600543 & -0.166881 & 4.553174 \\
\hline
\end{tabular}

Table 1. Registration results measured with respect to the implant: displacements in millimeters at the implant origin and tip, and rotation in degrees with respect to the implant's main axis.

In real situations where the image-based registration is actually performed, markers are not used. To evaluate the accuracy of registration, pre-operatively planned data must be transformed into the robot space with the computed registration and compared to the relevant data measured in the robot space. This subject is discussed next.

\section{$5 \quad$ Fusing Pre-operative Three-Dimensional Models With Intra-Operative Images: Qualitative Evaluation}

Let us suppose that we wish to show in one or several of the captured X-ray images, the position that the implant would have after surgery, assuming that the registration coordinates as determined in Section 3 are used to position and orient the robot with respect to the surgical plan. In this way, the surgeon 
will be able to see the clinical equivalent of post-operative X-ray images before the operation was actually executed. The following discussion applies as well if we wish to superimpose other three-dimensional shapes, such as a pre-operative femur bone model, or a model of a marker (screw) for marker-based registration.
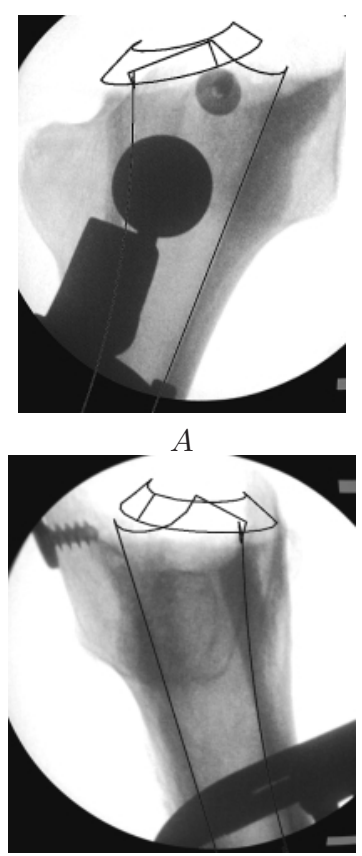

$D$

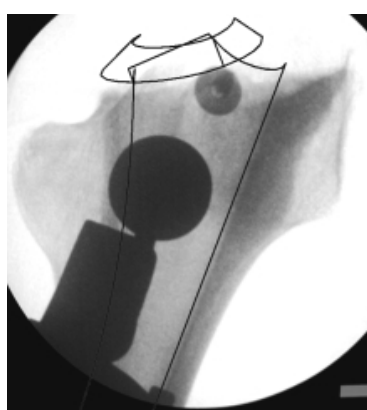

$B$

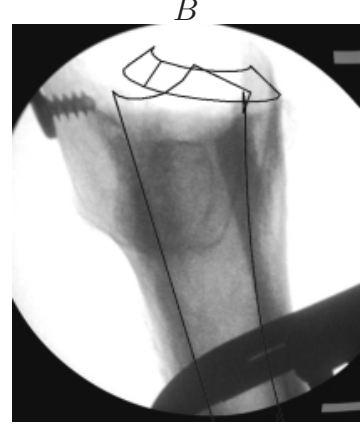

$E$
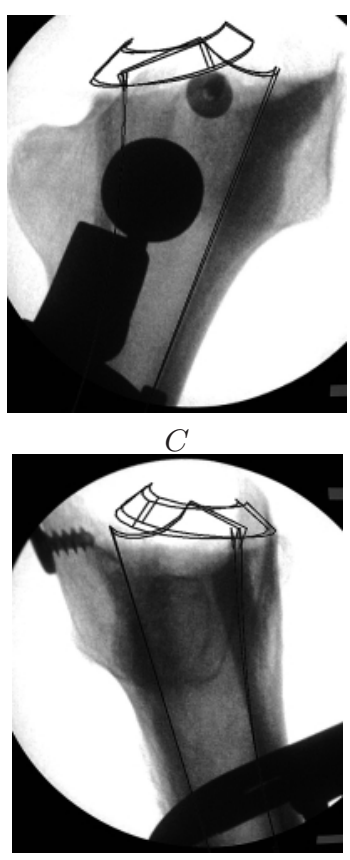

F

Fig. 5. A: fusion of X-ray and implant data using the marker-based registration. B: using the anatomy-based registration. D, E: idem on a second intra-operative $\mathrm{X}$-ray image, captured with a 60 degree rotation with respect to the previous image. C, F: comparing (superimposing) the marker-based registration with the image-based registration. The error in implant position is $1 \mathrm{~mm}$ or less (see Table 1). All images are corrected for distortion.

For a given X-ray image, using calibration information, we first determine a center of perspective (for instance, by finding a "best" point of intersection of several X-ray paths using Eqn 2, wherein all $\mathbf{p}_{\mathbf{i}}$ are at the origin and the resulting t gives the center of perspective), and use it to compute silhouette curves of the implant model as explained in Section 3 (for more detail, see [4]). Then, the method works independently of whether we wish to produce distortion-corrected images or distorted images: for each pixel of the X-ray image (original image pixels, or rectified image pixels) we determine an X-ray path knowing the pixel coordinates and the center of perspective. We then compute the distance from each X-ray path to the implant as discussed in Section 3. Finally, we convert the computed distances to gray-scale values. Various methods can be used to do this. To produce the images of Fig. 5 the following mapping was used: if the distance was less than $0.05 \mathrm{~mm}$, a gray-scale value of 0 was used, otherwise, if the 
distance was less than $0.1 \mathrm{~mm}$, a gray-scale value of 30 was used, otherwise, if the distance was less than $0.2 \mathrm{~mm}$, a gray-scale value of 60 was used, and otherwise, no change to the existing gray-scale value was done. This simple method allowed to avoid "aliasing" in the implant outline. One advantage of using distances to silhouette curves is that the resulting projection of the implant shows only the projected silhouette, which is sufficient to precisely indicate the position of the implant, but does not obscure any of the anatomy (see Fig. 5).

In Figure 6-A,E we show the pre-operative shapes that were used for fusion purposes. The implant was pre-operatively positioned accurately inside the CT data using the ORTHODOC ${ }^{\mathrm{R}}$ software. The implant model is shown projected inside (fused with) two distortion-corrected intra-operative X-ray images in Fig. 5. In Figs. 5-A,D, the implant model was registered using the marker-based method (current pin-based clinical protocol). In Figs. 5-B,E we use an image-based registration (without markers) obtained as in Section 3. Using Fig 5 we can reproduce a classical surgical planning method of superimposing acetate implant models onto film X-rays generally taken at right angles. Hence the images of Fig 5 should be easily interpretable by clinicians. In Figs. 5-C,F we superimpose the implant using both the marker-based and image-based registration. The difference in implant placement is no larger than $1 \mathrm{~mm}$, as reported in Table 1.

Fig.6 was obtained without correcting images for distortion. In Fig. 6-F, we superimpose the proximal marker onto a distorted X-ray image using the same registration transform as in Fig. 5-A. Fig. 6-A indicates that the pre-operative and intra-operative pin do not overlap exactly (the pin model, however, is longer than the physical pin): This confirms that the measured error is larger at the markers than at the implant. The error at the markers was measured in [20]. In Figs. 6-G,H, we also project the pre-operative femur model used for image-based (marker-less) registration.

\section{Conclusion}

Calibrated intra-operative X-rays can be used for registration and more. This paper described how to produce simulated post-operative X-rays. These images are particularly valuable because they are computed after the registration process: their validity is not subjected to a successful registration, but only to (1) the reliability and correct calibration of robotic devices executing the surgical plan. (As reported in [2,3] these are particularly reliable devices and processes.), and (2) the assumption that an incorrect registration cannot entirely compensate for an incorrect image calibration (for instance some inaccurate overlaps of the anatomy should be identifiable in fused images).

As illustrated in Fig. 5, we can thus provide post-operative simulations of the outcome of surgery before executing it. Furthermore, the simulations are presented in a standard clinical fashion, and are straightforward for a surgeon to interpret. Such images may help a surgeon decide to accept or reject a surgical plan or registration before any execution of the plan and thus may help prevent errors. 


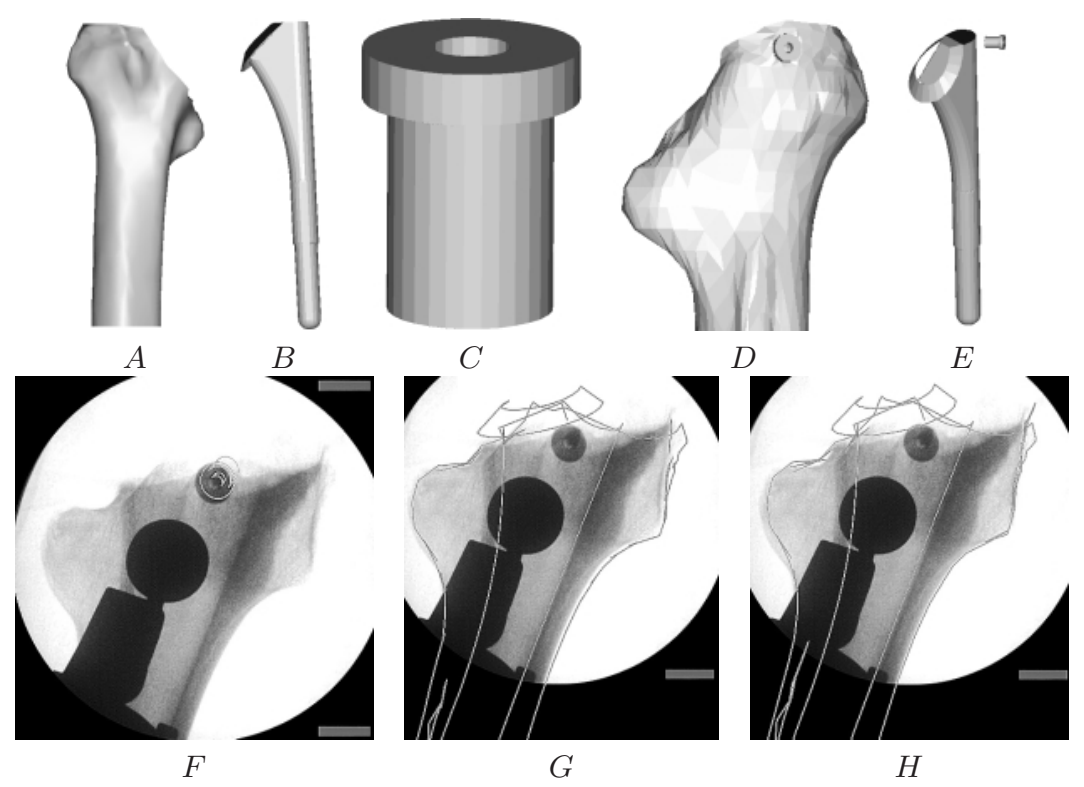

Fig. 6. A-E: Pre-operative model. A: CT-based proximal femur model B: implant CAD model (Depuy AML). C: simplified CAD model of fiducial pin $(8 \mathrm{~mm}$ diameter by $10 \mathrm{~mm}$ ). D: Femur and pin model registered in CT space. E: implant and pin model registered in $\mathrm{CT}$ space. F, G, and $\mathrm{H}$ are distorted images: the projection of shapes is distorted according to the image distortion model $\mathrm{F}$ : Superimposing the proximal pin (anatomy-based registration); the pin model is longer than the physical pin. G. H: superimposing femur and implant models. G: marker-based registration. H: anatomy-based registration.

By observing the simulated post-operative X-rays of total hip replacement surgery and studying the reported quantitative results, we are led to conclude that X-ray-to-CT registration using anatomical features provides comparable results to marker-based registration.

\section{Acknowledgments}

This work was partially funded by a Cooperative Agreement No. 70NANB5H1088 from the NIST Advanced Technology Program (ATP).

\section{References}

1. R.H. Taylor, L. Joskowicz, B. Williamson, A. Gueziec, A. Kalvin, P. Kazanzides, R. Van Vorhis, R. Kumar, A. Bzostek, A. Sahay, M. Boerner, and A. Lahmer. Computer-integrated revision total hip replacement surgery: Concept and preliminary results. Medical Image Analysis, 1999. to appear. 821 
2. R.H. Taylor, H.A. Paul, P. Kazanzides, B.D. Mittelstadt, W. Hanson, J.F. Zuhars, B. Williamson, B.L. Musits, E. Glassman, and W.L. Bargar. An image-directed robotic system for precise orthopaedic surgery. IEEE Transactions on Robotics and Automation, 10(3):261-275, 1994. 821, 830

3. B.D. Mittelstadt, P. Kazanzides, J. Zuhars, B. Williamson, P. Cain, F. Smith, and W.L. Bargar. The evolution of a surgical robot from prototype to human clinical use. In R.H. Taylor et al, editor, Computer-Integrated Surgery, pages 397-407. MIT Press, 1996. 821, 830

4. A. Guéziec, P. Kazanzides, B. Williamson, and R.H. Taylor. Anatomy-based registration of ct-scan and intraoperative x-ray images for guiding a surgical robot. IEEE Transactions on Medical Imaging, 17(5):715-728, oct 1998. 821, 822, 823, 825,829

5. S. Rudin, D.R. Bednarek, and W. Wong. Accurate characterization of image intensifier distortion. Medical Physics, 18(6), 1991. 1145-1151. 823

6. J.M. Boone, J.A. Seibert, W.A. Barrett, and E.A. Blood. Analysis and correction of imperfections in the image intensifier-TV-digitizer imaging chain. Medical Physics, 18(2), 1991. 236-242. 823

7. L. Launay, C. Picard, E. Maurincomme, R. Anxionnat, P. Bouchet, and L. Picard. Quantitative evaluation of an algorithm for correcting geometrical distortions in dsa images: Applications to stereotaxy. In Murray H. Loew, editor, Medical Imaging: Image Processing, volume 2434. SPIE, March 1995. 823

8. S. Schreiner, J. Funda, A.C. Barnes, and J.H. Anderson. Accuracy assessment of a clinical biplane fluoroscope for three dimensional measurements and targeting. In K.M. Hanson, editor, Medical Imaging: Image Display, volume 3031, pages 160166. SPIE, February 1997. 823

9. K.R Hoffmann, B.B Williams, J. Esthappan, S.Y.J Chen, J.D. Carroll, H. Harauchi, V. Doerr, G.N. Kay, A. Eberhardt, and M. Overland. Determination of 3D positions of pacemaker leads from biplane angiographic sequences. Medical Physics, 24(12), 1997. 1854-62. 823

10. J. Weese, T.M. Buzug, C. Lorenz, and C. Fassnacht. An approach to 2D/3D registration of a vertebra in $2 \mathrm{D}$ x-ray fluoroscopies with $3 \mathrm{D} \mathrm{CT}$ images. In Computer Vision and Virtual Reality in Medicine II - Medical Robotics and Computer Assisted Surgery III, volume 1205 of Lecture Notes in Computer Sciences, pages 119-128. Springer, 1997. 823

11. L. Tockus, L. Joskowicz, A. Simkin, and C. Milgrom. Computer-aided imageguided bone fracture surgery: Modeling, visualization, and preoperative planning. In MICCAI'98, Boston, MA, October 1998. 823

12. H. A. Martins, J. R. Birk, and R.B. Kelley. Camera models based on data from two calibration planes. Computer Graphics and Image Processing, 17:173-180, 1981. 823

13. G. Champleboux, S. Lavallée, P. Sautot, and P. Cinquin. Accurate calibration of cameras and range imaging sensors: the npbs method. In International Conference on Robotics and Automation, pages 1552-1557, Nice, 1992. IEEE. 823

14. S. Lavallee, R. Szeliski, and L. Brunie. Matching 3-D smooth surfaces with their 2-D projections using 3-D distance maps. In Geometric Methods in Computer Vision, volume 1570, pages 322-336, San Diego, July 25-26 1991. SPIE. 823

15. B. Lee. Stereo Matching of Skull Landmarks. PhD thesis, Stanford University, 1991. 823

16. F. Betting, J. Feldmar, N. Ayache, and F. Devernay. A new framework for fusing stereo images with volumetric medical images. In N. Ayache, editor, Computer 
Vision, Virtual Reality and Robotics in Medicine, volume 905 of Lecture Notes in Computer Science, pages 30-39, Nice, April 1995. Springer Verlag. 823, 825

17. A. Hamadeh, P. Sautot, S. Lavallée, and P. Cinquin. Towards automatic registration between ct and $\mathrm{x}$-ray images cooperation between $3 \mathrm{D} / 2 \mathrm{D}$ registration and 2D edge detection. In Proc. 2nd Int. Symp. on Medical Robotics and Computer Assisted Surgery, pages 39-46, Baltimore, MD, November 1995. 823

18. A. Liu, E. Bullitt, and S. Pizer. Surgical instrument guidance using synthesized anatomical structures. In Computer Vision and Virtual Reality in Medicine II Medical Robotics and Computer Assisted Surgery III, volume 1205 of Lecture Notes in Computer Sciences, pages 99-108. Springer, 1997. 823

19. R. Kumar and A.R. Hanson. Robust methods for estimating pose and a sensitivity analysis. CVGIP-IU, 60(3):313-342, 1994. 825

20. A. Guéziec. Registering intra-operative x-rays images with pre-operative data to simulate post-operative x-ray images. In 2nd Israeli Symposium on ComputerAided Surgery, Medical Robotics and Medical Imaging, May 1999. 830 